\title{
LES COLÊOPTĖRES PARASITES ACCIDENTELS DE L'HOMME (NOTE COMPLÉMENTAIRE)
}

\author{
Par Jean THÉODORIDĖS
}

Depuis la parution d'un travail antérieur (Théodoridès, 1948), j'ai pris connaissance d'autres travaux anciens et récents sur la question des coléoptères, parasites accidentels de l'homme (1), et je tiens à en exposer l'essentiel dans cette note complémentaire.

1. - Tout d'abord, il convient de donner la liste suivante de coléoptères pseudoparasites signalés par Hope et cités par Gervais et Van Beneden (1859). Etant donné l'ancienneté de ce traité et surtout celle des " cas » cités, l'on se gardera bien d'affirmer, sans réserves, l'authenticité de ceux-ci. Il a semblé cependant utile, pour rendre mon travail précédent plus complet, de donner ici cette liste où ne seront pas répétés les cas anciens déjà signalés (en particulier en ce qui concerne Tenebrio molitor L.).

\section{Carabidae.}

\section{Pseudoparasites cités par Hope:}

Sphodrus leucophtalmus L. 1 ex. rendu par l'estomac d'une femme, en Suède ; 1797 (Paykull, Upsal. Transact.).

\section{Dytiscidae.}

Dytiscus marginalis L. Larve trouvée en 1831 ou 1832 dans la poitrine d'une femme habitant le Middlessex, Angleterre (Hope, Trans. Ent. Soc. Lond.).

\section{Staphylinidae.}

Pxderus elongatus Fabr. Larve rejetée de l'estomac d'une femme, en Suède, 1796 (Paykull, Nov. Act. Upsal, VI, p. 115).

Oxyporus subterraneus Fabr. Larve rejetée de l'estomac d'une femme; en

Suède, 1798 (Paykull, loc. cit.).

Staphylinus politus Fabr. Id. 1797 (loc. cit.).

(1) Comme me l'a fait remarquer M. R.-Ph. Dollfus, il conviendrait pour être plus précis de distinguer parmi les coléoptères entre pseudoparasites (la majorité des cas signalés précédemment) et parasites accidentels (quelques cas très rares où le coléoptère se développe aux dépens de l'hôte : par ex. Necrobia sp. ct Niptus hololeucus) (Théodoridès, loc. cit.).

\footnotetext{
Ann. de Parasitologie, T. XXIV, $\mathrm{N}^{\circ s} 1-2 .-1949$, p. $110-115$.
} 
S. fuscipes Fabr. Nombreux ex. rejetés de l'estomac d'une femme, 1798. Id. (loc. cit.).

S. punctulatus Fabr. Quelques ex., même provenance, 1798 (loc. cit.).

\section{Dermestidae.}

Dermestes lardarius L. ; 3 cas cités:

1) Adulte rejeté de l'estomac d'une jeune fille (Bath, Angleterre, 1807, $D^{r}$ Chichester).

2) Adulte rejeté par l'anus d'un individu (Angleterre, Otto cité par Hope).

3) Autre adulte rejeté dans des conditions analogues.

Dermestes sp. 3 larves provenant de la poitrine d'une femme, en Angleterre (Martin Lister, Phil. Trans., 1665).

\section{Scarabaeidae.}

Geotrupes vernalis (sic). Estomac d'un enfant de 6 ans, en Suède, 1729

(Paykull, loc. cit. d'après Van Brommell).

Geotrupes sp. Suède, 1752 (id., d'après Rosen).

Melolontha sp. Estomac d'un enfant, en France, 1817 ou 1818 (Le Maout).

Plusieurs larves rejetées de l'estomac d'une femme, en France (le même, d'après Robineau Desvoidy).

\section{Tenebrionidae.}

Blaps mortisaga L. (voir plus loin) et Tenebrio molitor L.

Mordellidae (sic).

Mordella sp., Suède (Rosen).

Meloe proscarabaus (sic), adulte.

M. majalis (sic), id., rendu par l'intestin.

M. $s p$., rendu par l'estomac.

\section{Curculionidae.}

Balaninus nucum Fabr. Adulte provenant de la poitrine.

Autres cas : tube digestif et larves rendues par les voies urinaires.

2. - A propos de Tenebrio molitor L., il convient d'ajouter ce qui suit :

a. - Quatre cas anciens cités par Hope et mentionnés avec la liste de cet auteur donnée par Gervais et Van Beneden (loc. cit.) :

$1^{\circ}$ Ce coléoptère fut trouvé dans le nez (Allen in Bateman, 1811, loc. cit. in Théodoridès, 1948).

$2^{\circ}$ Id. chez une femme (Tulpius, 1652, loc. cit., ibid.).

$3^{\circ}$ Dans l'intestin, Ecosse ( ${ }^{r}$ Kollie).

$4^{\circ}$ Deux cas cités par Hope, non précisés.

b. - J. Leclercq (1948) vient de publier une enquête sur ce Ténébrionide considéré du point de vue parasitologique.

En plus des cas déjà cités dans mon travail précédent, cet auteur 
relate une note de Koldovsky (1930), qui a signalé à Olmütz (Tchécoslovaquie) un cas de conjonctivite granuleuse causée par l'encapsulement de $T$. molitor.

L'auteur tchèque décrit l'œil droit de la patiente, une femme de 41 ans, déjà soignée depuis quatre mois pour ce que l'on croyait être un trachome ; devant l'insuccès des traitements antitrachomateux, il pratiqua l'excision de la conjonctive et y découvrit des débris de $T$. molitor de 4 à $5 \mathrm{~mm}$. Après l'extraction de ceux-ci, l'œil fut soigné avec des gouttes à 3 p. 100 de noviforme et des bains d'oxycyanate de mercure à $1 / 500$. Trois semaines après, il ne restait aucune trace du processus granulaire.

La détermination de ces débris de coléoptère parait excessivement sujette à caution; vu leur petite dimension, il semble douteux qu'il s'agisse de $T$, molitor, mais il n'est pas à priori impossible qu'un coléoptère de petite taille ait pénétré accidentellement dans l'œil et de là se soit introduit dans la conjonctive.

Il n'est pas précisé dans ce travail s'il s'agissait de la larve ou de l'adulte.

3. - Renseignements complémentaires sur Blaps mortisaga L., parasite accidentel. - Blanchard (1890) signale plusieurs cas d'infestation accidentelle de l'intestin humain par ce Ténébrionide, et en particulier les observations de Cobbold (1877).

Cet auteur relate la découverte d'une larve de ce coléoptère dans les selles d'un enfant de 11 semaines. Le nourrisson était élevé avec du lait de vache toujours bouilli. Les symptômes observés furent les suivants : l'enfant toussa peu après sa naissance et, durant les cinq semaines précédant la surveillance médicale, * il avait une crise, une fois par semaine, apparemment de caractère convulsif, la figure devenant bleue durant les paroxysmes ». Il ne présentait aucune diarrhée et, tout au contraire, était constipé. Il fut traité avec de la poudre à base d'hydrargyrum et de la rhubarbe, qui agirent comme un antihelminthique.

Les caractéristiques ci-dessus ne confirmeraient nécessairement pas un cas de parasitisme accidentel si l'auteur n'en citait d'autres qui lui font admettre la vraisemblance du précédent.

Cas 1 (observé par Pickells, Thomson et Bellingham). - Une femme aurait évacué \& per anum 》 plus de 1.200 larves de $B$. mortisaga et même quelques adultes. Le cas pourrait passer pour invraisemblable, s'il n'avait été observé, entre autres, par le $\mathrm{D}^{\mathrm{r}}$ Bellingham, helminthologiste de Dublin bien connu.

Cas 2 et 3. - Seulement quelques larves étaient présentes dans l'intestin des patients (Patterson et Bateman).

Cas 4. - Signalé en 1841 par R. Cormack. 
Toujours d'après Cobbold, l'on pourrait trouver la référence d'autres cas en feuilletant d'anciens périodiques entomologiques.

Les trois premiers cas ci-dessus sont également mentionnés dans la liste de Hope, donnée par Gervais et Van Beneden (loc. cit.).

Il est difficile d'expliquer comment des larves de $B$. mortisaga peuvent pénétrer dans le tube digestif de l'homme ; cependant, dans le cas 1 de Cobbold, l'on découvrit que l'infestation provenait de ce que la malade était une démente qui avalait de la terre à des fins superstitieuses, et ceci de façon probablement répétée. Les larves de Blaps (comme celles de la plupart des Ténébrionides) se trouvent dans les endroits sombres et humides d'où elles ne sortent que la nuit.

Enfin, nous voyons dans Blanchard (loc. cit.) que c'est Hope qui a créé le terme de canthariasis (1) pour désigner la présince accidentelle de larves, nymphes ou mèmes adultes de coléoptères dans le tube digestif.

4. - Pour les cas de scarabiasis de l'Inde, il faut ajouter la référence d'une courte note anonyme, parue dans L'Indian Medical Gazette (1922), et signalant pour compléter les remarques parues dans le même périodique en 1919 et 1920, que Iyengar avait déterminé les Scarabéides incriminés comme étant Onthophagus bifasciatus (2).

5. - Enfin, j’ai eu connaissance de deux travaux anglais récents, mentionnant des coléoptères, parasites accidentels de l'homme :

Le premier est une note de Donisthorpe (1942) signalant que d'après un docteur du "Royal Cancer Hospital », une femme aurait rejeté, avec son urine, deux exemplaires de Atheta longuiscula Gr. et un de Stilicus affinis Er. (Staphylinidæ). Comme le fait remarquer l'auteur, la présence de ces coléoptères adultes dans la vessie humaine paraît absolument impossible, mais il se demande d'où ils pouvaient provenir, car ils furent trouvés à une époque où normalement ils hivernent et où il faut vraiment un coléoptériste expert pour les découvrir. T. Bainbrigge Fletcher fait remarquer, à la suite de la note de Donisthorpe, que ce « cas » est d'autant plus sujet à caution du fait que deux espèces différentes y étaient incriminées.

Personnellement, nours ne croyons aucunement à l'authenticité de

(1) Il convient de faire remarquer ici que le terme de « canthariasis » ayant été créé par un auteur anglais, on devrait dire, en français, "canthariase », si l'on considère que la traduction du terme anglais \& myiasis 》 est « myiase ». Ceci s'applique aussi au terme de « scarabiasis ».

(2) Je remercie ici le $\mathrm{D}^{r} \mathrm{~J}$. Balazuc qui m'a communiqué cette référence.

Ans. de Parasitologie, t. XXIV, $\mathrm{N}^{\mathrm{os}} 1-2 .-1949$.

8. 
ce cas et supposons que les coléoptères hivernaient dans le bâtiment où se trouvaient les récipients destinés à collecter les urines et qu'ils pénétrèrent dans ceux-ci.

Le second travail est une note de Sharpe (1947), signalée aussi par Donisthorpe (1948), mentionnant un cas de canthariasis dù à Ptinus tectus Boield. (Ptinidx) ; une femme de 40 ans, à la clinique du «Queen Mary's Hospital 》 de Londres, prétendit avoir rejeté per anum trois larves de $5 \mathrm{~mm}$. vivantes, qui furent déterminées par le Dr F. I. Van Emden comme étant celles du coléoptère mentionné ci-dessus. L'auteur rapporte que deux semaines auparavant, la malade avait souffert de fréquentes attaques de diarrhée et de constipation ; elle avait remarqué des taches de sang dans ses selles, et c'est en examinant celles-ci avec attention qu'elle y trouva les larves. L'auteur insiste également sur le fait que la malade était très propre, intelligiente et donc digne de foi.

Traitement. - De la paraffine liquide donnée à petite dose (1 once), la nuit, pendant sept jours, amena une guérison totale.

Sharpe affirme l'authenticité du cas et suggère que les oufs de ce coléoptère ont une enveloppe chitineuse qui leur permet le passage à travers l'estomac et que les larves pourraient éclore dans la partie plus alcaline de l'intestin. Il est à remarquer qu'il commet deux erreurs : $a$ ) lorsqu'il emploie le terme de myiase réservé aux larves de diptères ; $b$ ) lorsqu'il dit que les larves de $P$. tectus sont insectivores (insectivorous larvæ) ; il a sans doute voulu simplement dire qu'il s'agissait de larves d'insectes (insect larvæ).

Van Emden, dans une lettre au British Medical Journal (où avait paru le travail précédent), critique les assertions de Sharpe quant à la possibilité pour les larves de $P$. tectus de traverser sans encombre le tube digestif humain (1947).

Ce dernier auteur suggère que les larves incriminées se trouvaient dans le récipient où furent recueillies les selles et critique Sharpe de ne pas avoir donné de description de celui-ci où elles auraient pu tomber d'un matelas, drap ou coussin. De plus, le revêtement de l'œuf des Ptinides est très mince et ne pourrait résister à la pepsine et l'acide chlorhydrique de l'estomac.

Pour vérifier ce dernier fait, Van Emden a traité des œufs, de jeunes larves et des larves àgées de $P$. tectus avec du suc gastrique artificiel. Les exemplaires furent mélangés à du pain mâché et l'expérience se fit à une température de $36-38^{\circ}$, pendant trois heures. Ce matériel fut alors lavé avec de l'eau distillée et observé sous le binoculaire : les larves jeunes et âgées étaient mortes, mais non désintégrées ; celles prêtes à éclore avaient été tuées dans les œufs, 
dont la paroi n'offrirait done aucune protection. L'auteur souligne que $P$. tectus vit dans lés matières organiques sèches, d'une part, et dans les céréales, poivre de Cayenne, cacao, biscuits de mer, de l'autre. Ce coléoptère serait adapté pour vivre dans un milieu contenant de 5 à 15 p. 100 d'humidité, et il parait difficile d'admettre que les larves puissent supporter celle de l'intestin (plus de 90 p. 100), d'autant plus qu'expérimentalement seul un faible pourcentage de larves survit à 40 p. 100 d'humidité. Mais d'après Van Emden, la cause principale empêchant la vie des larves de $P$. tectus dans le tube digestif serait la température de celui-ci ; d'après des expériences de laboratoire, les œufs de Ptinides ne peuvent éclore au-tessus de $28^{\circ}$ et les larves aux premiers stades ne peuvent survivre à une température de $29^{\circ}$.

Néanmoins, le cas signalé par Sternberg (cf. Théodoridès, loc. cit.) semble absolument authentique, parce que contrôlé expérimentalement, et bien que les intéressantes remarques de Van Emden montrent qu'au laboratoire les Ptinides ont des préférenda écologiques bien délimités, il ne semble pas à priori impossible que dans certains cas des coléoptères de cette famille ne puissent supporter les conditions d'existence très particulières présentées par le tube digestif de thomme.

\section{BiBLIOGRAPHIE}

1890. - Br.avchard (R.). - Traité de Zoologie Médicale, 2 vol., Baillière, Paris. 1922. - (Anonyme), - Intestinal Scarabiasis. Ind. Med. Gaz., LVII, 227-28.

1877. - CoввоL. (T. S.). - On Blaps mortisaga as a human parasite. Brit. Med. J., $1877, \mathrm{I}, 420$.

1942. - Doxisthonpe (H.). - A strange story. Entom. Rec. J. Variat, LIV, 79. 1948. - Doxisthorpe (H.). - Some notes on Ptinus tectus Boield. (Ptinida). Ibid., LX, 2, 119.

1947. - Emoex (F. I. van). - Intestinal Myiasis. Brit. Med. J., 1947, I, 350-51. 1942. - Fi.etcher (T. Bainbrigge). - Notes on the above \& strange story \$. Entom. rec. J. Variat., LIV, 79-80.

1859. - Gervais (P.) et vas Beneden (P. J.) . - Zoologie Médicale, 2 vol., Baillière, Paris.

1930. - KoLpovskx (K.). - Jednostrannà conjunctivitis granulosa zpǔsobena upouzdrenin Trupu Moucneho Broùcka (Tenebrio molitor). Bratislav. Lekarsk. List., 10, 1, 44-46.

1948. - Leccence (J.), - Enquête parasitologique sur le ver de farine Tenebrio molitor L. Lambill., 48 année, 5-6, 43-48 ; 7-8, 52-58.

1947. - Sharpe (D. S.). - An unusual case of intestinal myiasis. Brit. Med. J., 1947 , I, 54, 1 fig.

1948. - Th⿱㇒́oponidè̀s (J.). - Les coléoptères parasites accidentels de l'homme. Ann. Parasit., XXIII, 348-63.

Museum National d'Histoire Naturelle de Paris. 